\title{
A Nonlinear Model of Visual Information Processing Based on Discrete Maximal Overlap Wavelets
}

\author{
Hitoshi ARAI* \\ Graduate School of Mathematical Sciences, The University of Tokyo, \\ Komaba 3-8-1, Meguro-ku, Tokyo 153-8914, Japan \\ E-mail: h-arai@ms.u-tokyo.ac.jp
}

Received April 6, 2005; final version accepted May 9, 2005

\begin{abstract}
The purpose of this paper is to give a new computational model of early visual information processing, and to simulate by using the model the occurrence of visual illusions. The model proposed in this paper is constructed as a maximal overlap biorthogonal wavelet filter bank equipped with a nonlinear processing modeled after "contrast induction" effect (for the definition, see Section 3). This model provides good computer simulations of the occurrence of many lightness illusions such as the Mach band, the Hermann grid, the Chevreul illusion, and other related illusions. Moreover, also the café wall illusion is studied by using the model.
\end{abstract}

KEYWORDS: wavelet, lightness illusion, contrast induction

\section{Introduction}

As is well known, a considerable number of studies have been conducted on lightness illusions. In particular, over the past few decades, the concern with mathematical methods such as neural network analysis and bandpass filtering has been growing. In this paper I propose a new nonlinear data processing modeled after the contrast induction (for the definition, see Section 3), and construct a computational nonlinear system by incorporating both this processing and a maximal overlap biorthogonal wavelet filter bank. Further by using this system, several computer simulations of the occurrence of visual illusions are presented. Before going to the main argument, I would like to explain why the wavelets are used in this paper. From a mathematical viewpoint, wavelet filters have two striking features: one of them is that wavelets form the so-called multiresolution analysis, and another is that wavelets can make a perfect reconstruction filter bank. The multiresolution structure is regarded as a mathematical analogue of the multi-channels hypothesis originated by Campbell and Robson [5] (for more sophisticated study, see De Valois [9]). Therefore for studying visual information processing it is natural to use wavelets. It is known that the multi-channels can be simulated also by using Gabor filters or oriented DOG filters (see Blakeslee and McCourt [4]), however by using wavelet filter banks one can easily construct the multiresolution analysis. In addition, the perfect reconstruction property of wavelet filter banks is very useful in order to construct nonlinear processings for computer simulations of the occurrence of visual illusions. In fact, if the system is not perfect reconstruction, one can not judge whether the outputted illusory figures in the simulation are due to the nonlinear processing or to the failure of the system itself.

It should be noted also that a wavelet filter bank consists of two phases: one of them is the analysis phase and another is the synthesis phase. The former represents in terms of mathematics that the striate cortex decomposes visual information into several subband signals. However it is believed that the latter does not exists in the brain (cf. [29]). In this paper the synthesis phase is used only for the technical purpose of visualizing what happens on visual information after the nonlinear processing.

This paper is organized as follows. Section 2 is devoted to describe which wavelets are used in this paper. In Section 3, I introduce a mathematical formulation of the contrast induction and a computational model of visual information processing. Section 4, which is the main part of this paper, is devoted to describe the result of simulations for several lightness illusions: the Mach band, the Hermann grid, the Chevreul illusion, and other related illusions. Moreover, also the café wall illusion and a natural image are analyzed.

Some similar simulations for lightness illusions were announced in Arai [1] without mathematical discussions and the description of the mathematical structure of our nonlinear system.

Acknowledgement. The author is grateful to Mrs. Shinobu Arai for her considerable assistance and insightful comments. Without her assistance he could not finish this paper. 


\section{Wavelets}

So far many wavelets have been established by many applied mathematicians for various aims. This section is devoted to describe which wavelets are used in this paper. Among many wavelets, I adopt compactly supported biorthogonal wavelets introduced by Cohen, Daubechies and Feauveau [6]. More specifically, I use their wavelets in the case of $k=4, N=4$ and $\widetilde{N}=4$ (for the terminology, see [6]), however employ the analysis filters (resp. the synthesis filter) for synthesis (resp. analysis), because as is pointed out by A. Cohen, the analysis (resp. synthesis) filters in [6] have the useful properties for synthesis (resp. analysis). For further details, see [19]. The reason why their biorthogonal wavelets are adopted in this paper is that the graphs of the wavelets are very similar to the quantitative simple cell receptive field profiles depicted in [9, p. 122]. Furthermore their supports lie in compact sets as the extent of the receptive fields in the cortex is finite. Since these wavelets are one-dimensional, as usual way two-dimensional wavelets are defined by tensor products of these one-dimensional wavelets. Then the tensor products cover three main directions: horizontal, vertical and diagonal. As is well known by the physiology, simple cells have also selectivity for various directions. However because the main task in this paper is to find mathematical description of "contrast induction" effect, we concentrate our attention only to these three main directions for the sake of the technical simplicity. Nevertheless, fortunately it is enough to take only these directions into consideration in order to analyse illusory figures which will be discussed in this paper, because they have relatively simple geometric structure.

There is one other thing that should be noted. The filter bank in this paper is not maximally decimated, but is maximally overlapped, namely, it is the so-called stationary wavelet filter bank introduced by R. R. Coifman, D. L. Donoho, G. P. Nason and B. W. Silverman et al. (see [7, 21, 23]) The reason why I adopt the stationary wavelets is that it is robust with respect to nonlinear processing, because the subband signals are highly redundant.

Now in order to describe our results in later sections, let us here introduce some notation. Since the stationary wavelet filter bank has a pyramid structure, it possesses several stages. At the first stage the filter bank decomposes an input signal $x$ into four type of subband signals: the level 1 approximation and level 1 horizontal, vertical, and diagonal details. We denote them by $x_{1}^{a}, x_{1}^{h}, x_{1}^{v}$, and $x_{1}^{d}$, respectively. At the second stage the filter bank decomposes the signal $x_{1}^{a}$ into four subband signals: the level 2 approximation and the level 2 horizontal, vertical, and diagonal details. We denote them by $x_{2}^{a}, x_{2}^{h}, x_{2}^{v}$, and $x_{2}^{d}$, respectively. By the same way $k$ th level subband signals $x_{k}^{a}, x_{k}^{h}, x_{k}^{v}$, and $x_{k}^{d}$, $k=3, \ldots, L$, are obtained, where $L$ is the number of channels. In this paper $L$ is set to 8 because of convenience for computer simulation. In what follows, for a two dimensional signal $x=(x[m, n])_{m, n}$, let $\operatorname{abs}(x)=(|x[m, n]|)_{m, n}$. It is called the magnitude of the signal $x$.

\section{Nonlinear Processing}

The purpose of this section is to introduce a nonlinear processing modeled after the phenomenon which is called the contrast induction effect. Let us begin with describing the contrast induction effect. The reader can see this effect in Fig. 1: the stripe in the middle of each image in Fig. 1 has physically the same luminance, however our perception is that the luminance of the stripe in the middle of (c) is lower than one of the stripe of the middle of (b) or (a). The difference between these images is that the stripe in (a) is surrounded by a high contrast grating, but the stripe in (b) is surrounded by a low contrast grating, and in (c) the surround has a uniform luminance. This is called the contrast induction. In this paper I present a new mathematical model of this phenomenon.

In order to simulate the contrast induction, one need to find a mathematical operator which enhances small values of a detail if the energy of the detail is small, and inhibits small values of a detail if its energy is large. Our idea to define such an operator is as follows. N-shaped functions enhance small values, on the other hand S-shaped functions inhibit small values. Therefore it is desirable to find a function $f(x, \alpha)$ with a parameter $\alpha$ satisfying that the function $f(\cdot, \alpha)$ metamorphoses smoothly from $\mathrm{S}$-shape into $\mathrm{N}$-shape depending on the parameter $\alpha$. Moreover it is convenient for computation that for all $\alpha$ the functions $f(\cdot, \alpha)$ are defined on a common bounded interval, and that for each $\alpha$ both the initial point and the terminal point of the graph of $f(\cdot, \alpha)$ are identical. In order to define such a family of functions, let us begin to deform the sigmoid function $\varphi(x)$ with the condition $\varphi(0)=0$ defined by

(a)

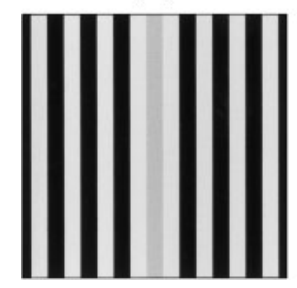

(b)

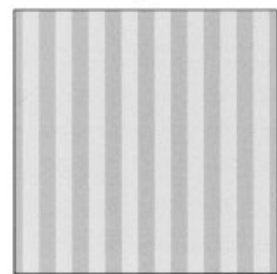

Fig. 1. (c)

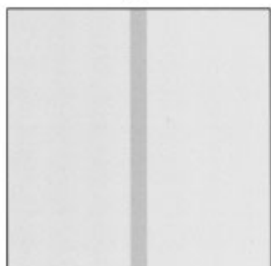




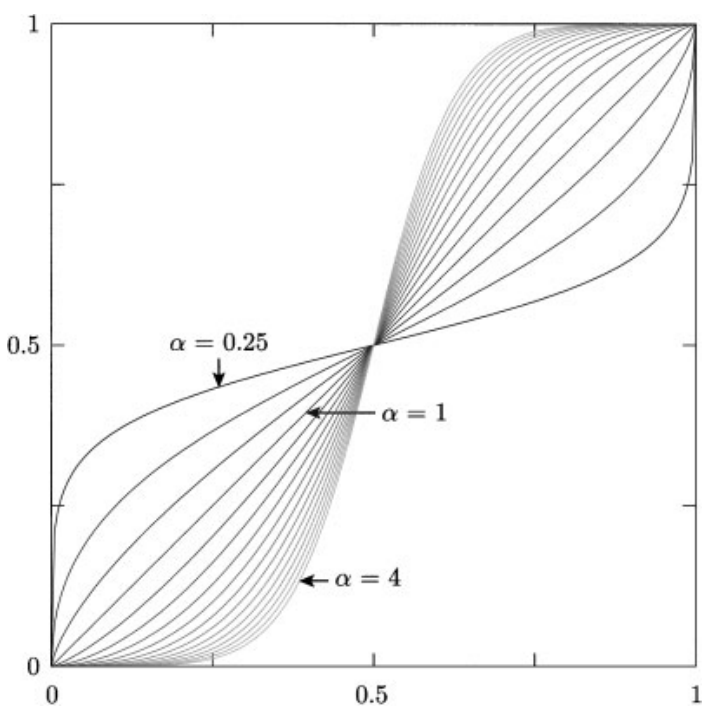

Fig. 2. If $\alpha>1, s(x, \alpha)$ is S-shaped, if $\alpha=1, s(x, \alpha)=x$, and if $\alpha<1, s(x, \alpha)$ is N-shaped.

$$
\varphi(x)=\frac{2 e^{x}}{1+e^{x}}-1
$$

Since the sigmoid function is defined on the real line, it must be modified to a function defined on a bounded interval. A way of accomplishing such modification is to composite the function $\varphi(x)$ and an appropriate function $\psi$ from a bounded interval onto the real line. As a function satisfying all these demands, I propose the following

$$
S(x, \alpha)=\varphi(2 \alpha \operatorname{arctanh}(x)) .
$$

We call this function an SN-function. The SN-function is very useful because if $\alpha>1, \alpha=1$, and $\alpha<1$, then $S(x, \alpha)$ represents the sigmoid function, a linear function and $\mathrm{N}$-shaped function, respectively. Figure 2 depicts the graphs of normalized form

$$
s(x, \alpha)=\frac{S(2 x-1, \alpha)+1}{2}, 0 \leq x \leq 1
$$

of the SN-function for $\alpha=0.25+0.25 n, n=0,1, \ldots, 15$ (Fig. 2). In what follows we call also the normalized form $s(x, \alpha)$ an $\mathrm{SN}$-function. By easy calculation we have that

$$
s(x, \alpha)=\frac{x^{\alpha}}{x^{\alpha}+(1-x)^{\alpha}}, 0 \leq x \leq 1,0<\alpha .
$$

To summarize, the $\mathrm{SN}$-function satisfies the following property: if $\alpha$ is smaller than 1 , then $s(x, \alpha)$ enhances all small values, on the other hand if $\alpha$ is greater than $1, s(x, \alpha)$ inhibits small values. Now the desired nonlinear processing can be achieved by using the SN-function. To be exact, the processing is defined for each level by operating the SNfunction to the magnitude signals $a b s\left(y_{k}^{\varepsilon}\right)$ of the signals $y_{k}^{\varepsilon}(c f$. Fig. 3) which are obtained by a normalization of the values of the details $x_{k}^{\varepsilon}, \varepsilon=h, v, d$ into the unit interval $[0,1]$. Here the parameter $\alpha$ is taken such that $\alpha$ is small (resp. large) if the energies of the details at each level are small (resp. large). For example, in this paper I determine experimentally the parameter as follows

$$
\alpha\left(y_{k}^{\varepsilon}\right)=\left\{\begin{array}{ll}
0.8\left\|y_{k}^{\varepsilon}\right\|_{l^{2}}^{1 / 8}+0.4, & \text { if } \varepsilon=h, v \\
0.4\left\|y_{k}^{\varepsilon}\right\|_{l^{2}}^{1 / 4}+0.8, & \text { if } \varepsilon=d
\end{array}, k=1, \ldots, 8,\right.
$$

where $\|\cdot\|_{l^{2}}$ is the usual normalized $l^{2}$ norm, that is, $l^{2}$ norm with respect to the counting probability measure. The reason why the parameter $\alpha$ is defined in different ways depending upon $y_{k}^{\varepsilon}$ is diagonal or not (i.e. $\varepsilon=d$ or $\varepsilon=h$, v) is that the visual information processing for the diagonal direction is not same as that for the horizontal and vertical directions. For instance, the strength of the Hermann grid illusion whose grid is presented diagonally is weaker than the original one (see Spillmann [26]). As noted in [26], it is due to the influence of orientation-sensitive cells in LGN or in V1. Taking these facts into consideration, in this paper the parameter $\alpha$ is defined by (3). Consequently, from the behavior of the SN-function with this parameter it follows that our nonlinear processing satisfies the mathematical conditions which are characteristic features of the contrast induction. In this paper we call the paprameter $\alpha\left(y_{k}^{\varepsilon}\right)$ the $k$ th horizontal (resp, vertical, diagonal) contrast exponent for $y_{k}^{\varepsilon}$ when $\varepsilon=h$ (resp. $\varepsilon=v, \varepsilon=d$ ).

For simplicity, we call the nonlinear system constructed in this section the SNW system. 


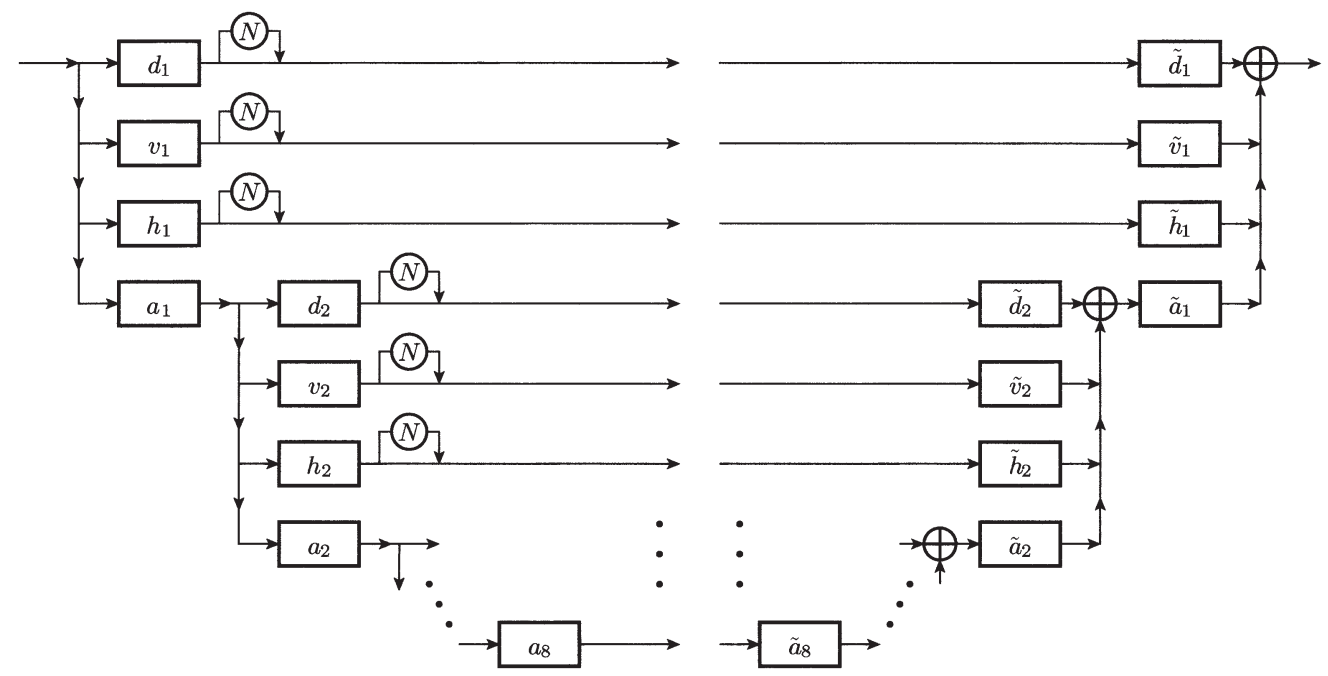

Fig. 3.

(a) Input

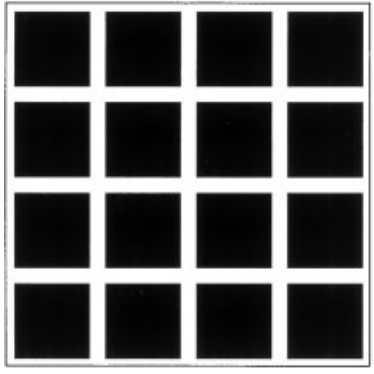

(b) Output

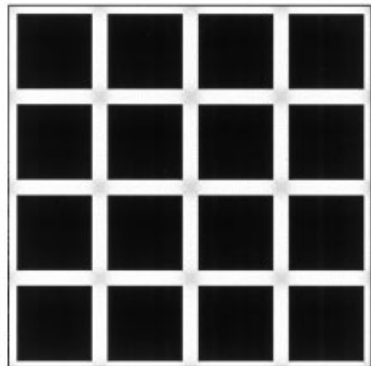

(c) Profile of (b)

Fig. 4. Left: the Hermann grid. Middle: the output image, Right: the profile of (b).

\section{Computer Simulations}

In this section I show computer simulations of the occurrence of visual illusions by using the SNW system described in the previous section, and explain mathematically how the visual illusions appear. Let us begin with a typical lightness illusion, the Hermann grid illusion.

\subsection{The Hermann grid illusion and its variations}

The Hermann grid is shown in Figure 4(a). In this image, small gray spots are perceived at the intersections of white streets, although there are physically no such spots. (Note that the illusion disappear in foveal vision.) Classically it was thought that the illusion is caused by lateral inhibition in the retina (Baumgartner, 1960). However also some evidences of a postretinal contribution to the illusion were reported (see [26]). The result of our simulation is shown in Fig. 4(b), that is, (b) is the image outputted when Fig. 4(a) is inputted to the SNW system. For the sake of displaying, Fig. 4(b) is scaled such that [min, max] is mapped to $[0,255]$, where min is the minimum of the luminance of the outputted image and max is its maximum. From now on, in this paper we say that an image is scaled for displaying if it is scaled such that [min, max] for the image is mapped to [0,255]. Figure 4(c) illustrates the profile of the luminance of the output along the horizontal white road through the center of the image. As is evident from Fig. 4(b) and (c), the output image contains physically dark spots at each intersections of white bars. Let us describe how the SNW system produces dark spots. Since the Hermann grid pattern consists of horizontal and vertical segments, the energies of horizontal and vertical details are bigger than ones of diagonal details. Hence the horizontal contrast exponent and vertical one are greater than 1 if the level is between 3 and 6, whereas all diagonal contrast exponents are smaller than 1 (see Fig. 5). Moreover since those values among $a b s\left(y_{j}^{h}\right)$ which are concerned with the middle areas of two adjacent crosses are greater than 0.5 (see Fig. 6(a)), they are enhanced by the $\mathrm{SN}$-function. On the other hand, because those values among $a b s\left(y_{j}^{h}\right)$ which are related to crosses are smaller than 0.5 , the $\mathrm{SN}$-function attenuates them. The behavior of vertical wavelet coefficients $y_{j}^{v}$ is same as the horizontal one. The diagonal wavelet coefficients are different from horizontal and vertical ones. The diagonal contrast exponents are smaller than 1, and as shown in Fig. 6(b), all values of $a b s\left(y_{j}^{d}\right)$ are smaller than 0.5 . Moreover the values of $a b s\left(y_{j}^{d}\right)$ are very small except a neighborhood of each cross. Therefore near the middle of two adjacent crosses, the values of $a b s\left(y_{j}^{d}\right)$ are strongly enhanced, but near each cross the values of 

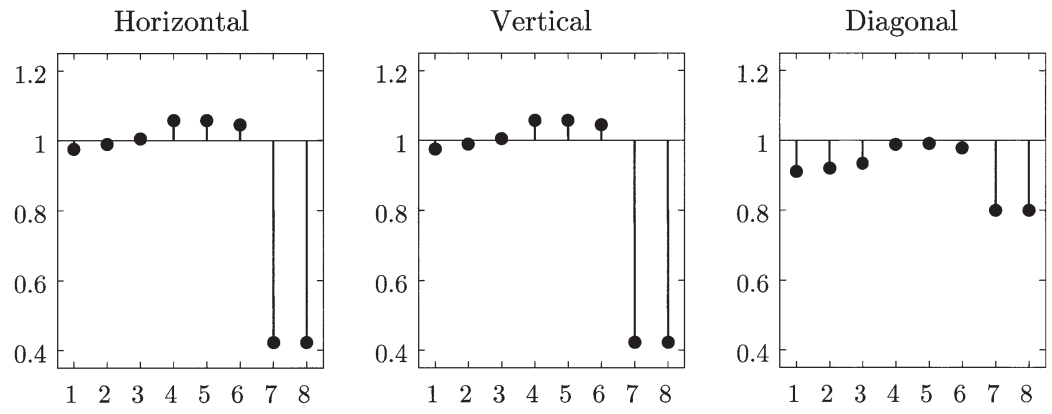

Fig. 5. Contrast exponents for the Hermann grid pattern.

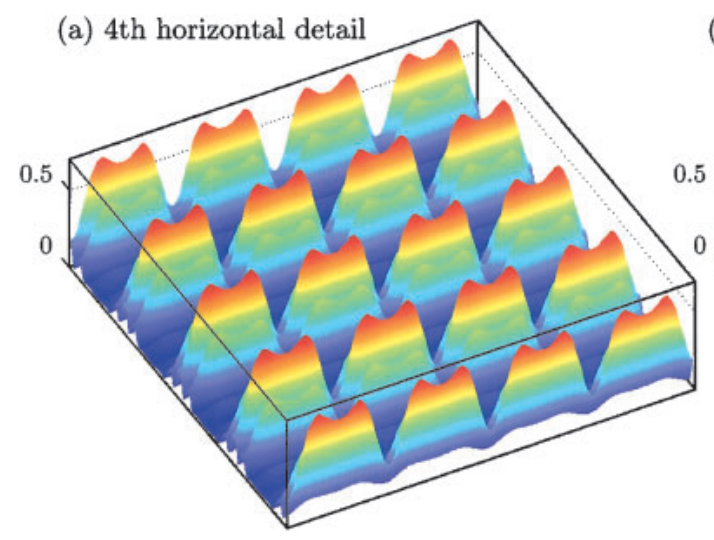

(b) 4th diagonal detail

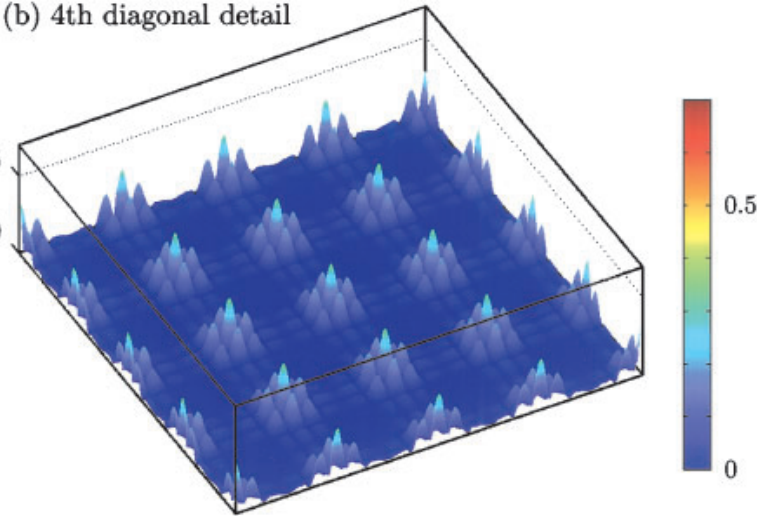

Fig. 6.
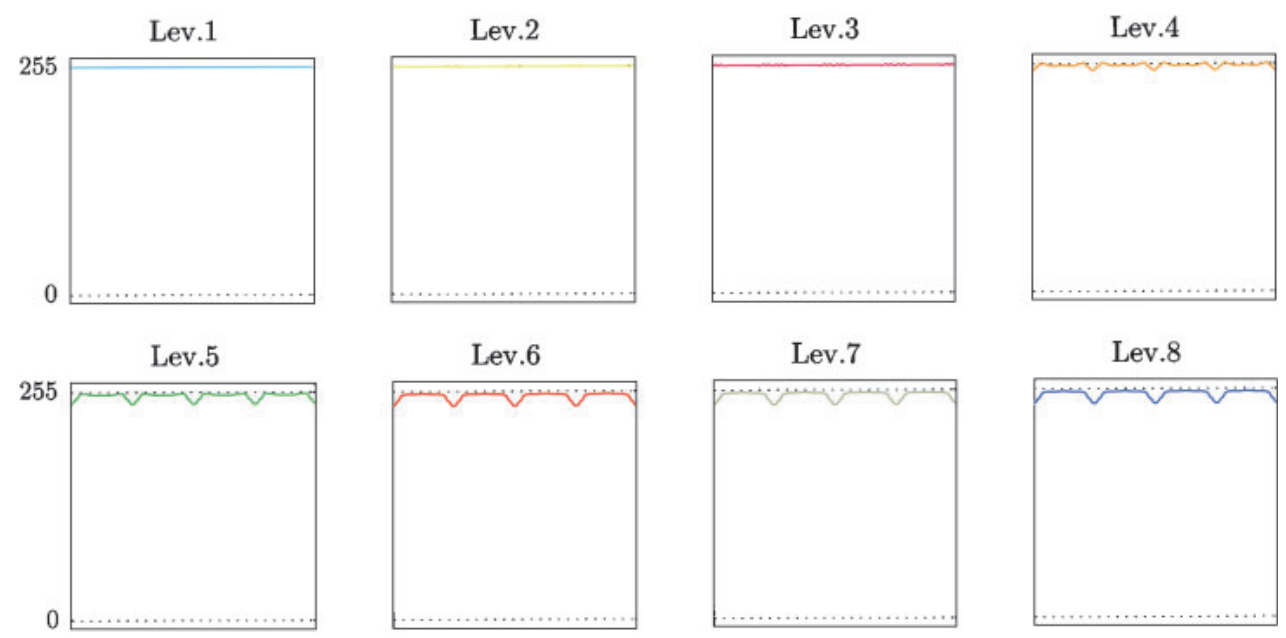

Fig. 7.

$a b s\left(y_{j}^{d}\right)$ are not so strongly enhanced. Thus the dark spots appear at the crosses of white streets.

Figure 7 shows the profiles of the $k$ th level output $\widetilde{x}_{k}$ defined by

$$
\widetilde{x}_{k}=\sum_{j=1}^{k}\left(\widetilde{h}_{j} * F_{j}\left(x_{j}^{h}\right)+\widetilde{v}_{j} * F_{j}\left(x_{j}^{v}\right)+\widetilde{d}_{j} * F_{j}\left(x_{j}^{d}\right)\right)+\widetilde{a}_{k} * x_{k}^{a}
$$

$(k=1, \ldots, 8)$ where $*$ is the convolution operator, $F_{j}\left(x_{j}^{\varepsilon}\right)$ denotes the signal which is obtained by action to the detail $x_{j}^{\varepsilon}$ of the nonlinear processing described in Section 3, and $\widetilde{a}_{j}, \widetilde{h}_{j}, \widetilde{v}_{j}$, and $\widetilde{d}_{j}$ are reconstruction filters for approximation, horizontal, vertical and diagonal details respectively.

In 1971 Spillmann and Levine pointed out an evidence for postretinal contribution to the Hermann grid illusion. They asserted that the Hermann grid illusion is weakened when the grid is presented diagonally ([26]). Figure 8(b) is the result of our simulation for the original image, Fig. 8(a). (Figure 8(b) is scaled for displaying.) Because the contrast exponents for the diagonal part are defined weaker than ones for horizontal or vertical parts, the SNW system can simulate Spillman and Levine's phenomenon. Figure 8(c) illustrates the profile of the luminance of Fig. 8(b) along the 
(a) Input

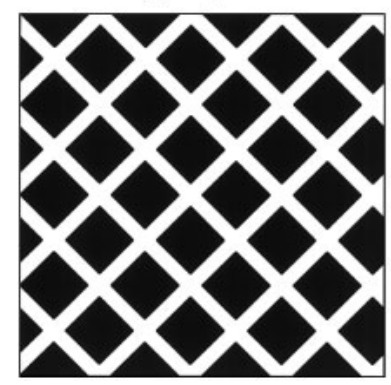

(a) Input

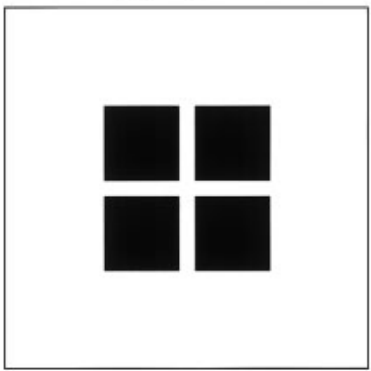

(b) Output

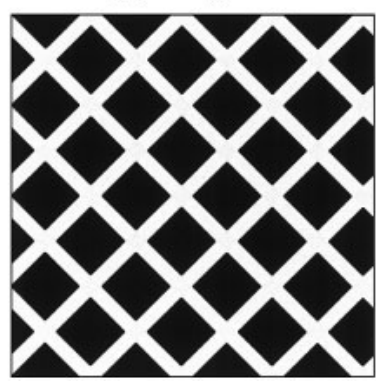

Fig. 8. (c) Profile of (b)

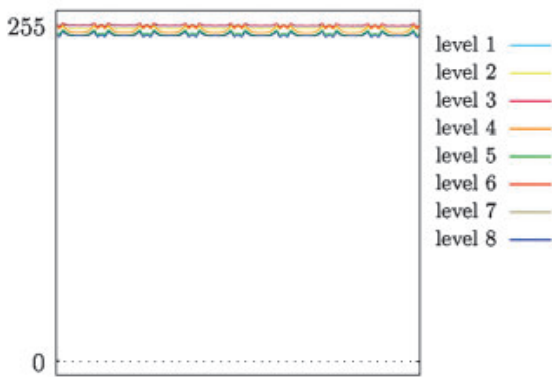

(c) Profile of (b)

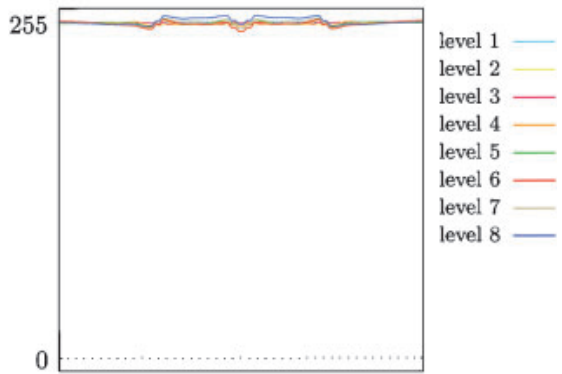

Fig. 9.
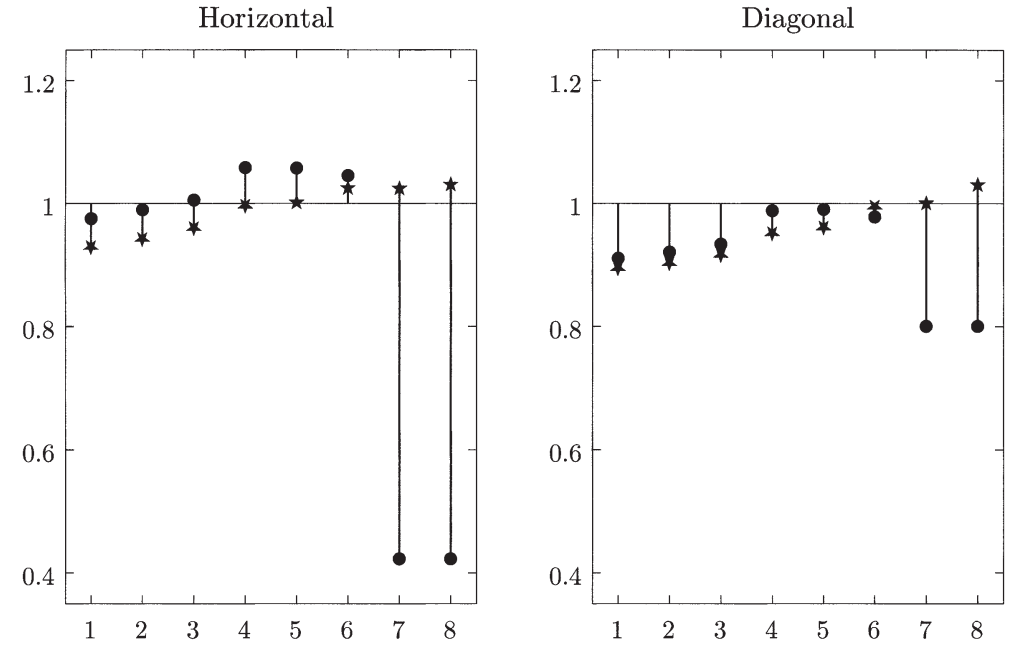

Fig. 10. Contrast exponents for Hermann grid and Wolfe's Hermann grid. '•’: grid, ' $\star$ ': Wolfe's grid.

diagonal white road through the center of the image (b).

In 1984 Wolfe observed that the strength of the Hermann grid illusion is depending on the number of intersections: if the number increases, then the strength of the illusion increases (see [28]). We can simulate also this phenomenon. The result of our simulation is shown in Fig. 9(c) and (b) which is scaled for displaying. The reason why the illusion of Fig. 9 becomes weaker is that the energies of details at almost all levels are smaller than ones of the original Hermann grid. In fact, we have that $1<\alpha_{k}^{\prime}<\alpha_{k}$, for all $k$ except 7 and 8 , where $\alpha_{k}$ and $\alpha_{k}^{\prime}$ are the contrast exponents for the original Hermann grid and for the left image in Fig. 9 respectively (see Fig. 10). Therefore the SNW system produces Wolfe's phenomenon.

\subsection{The Chevreul illusion and a nonlinear effect}

The purpose of this section is to study the Chevreul illusion (see Fig. 11). It consists of steps of homogeneous luminance, but the bands seem to be darker at one edge than at the other. The result of the simulation is shown in Fig. 12. The left image of Fig. 12 is the output and the right figure is the graph of the profiles of all $k$ th level outputs $\tilde{x}_{k}$ (see (4) for the definition). For reader's convenience, we depict the profiles of $\tilde{x}_{k}$ 's separately in Fig. 13. The reason why the SNW system can simulate this illusion is as follows: by the orientation-selectivity of the wavelets which are 


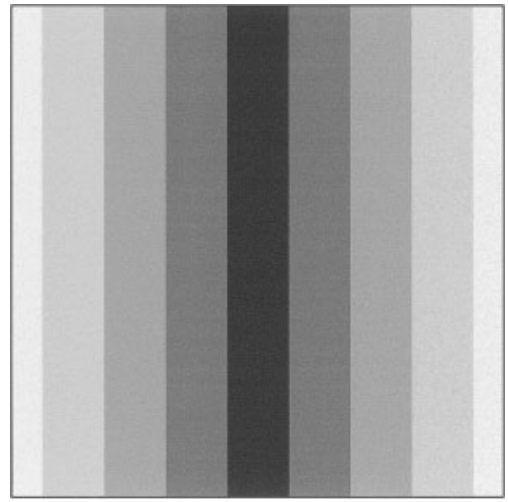

Fig. 11.
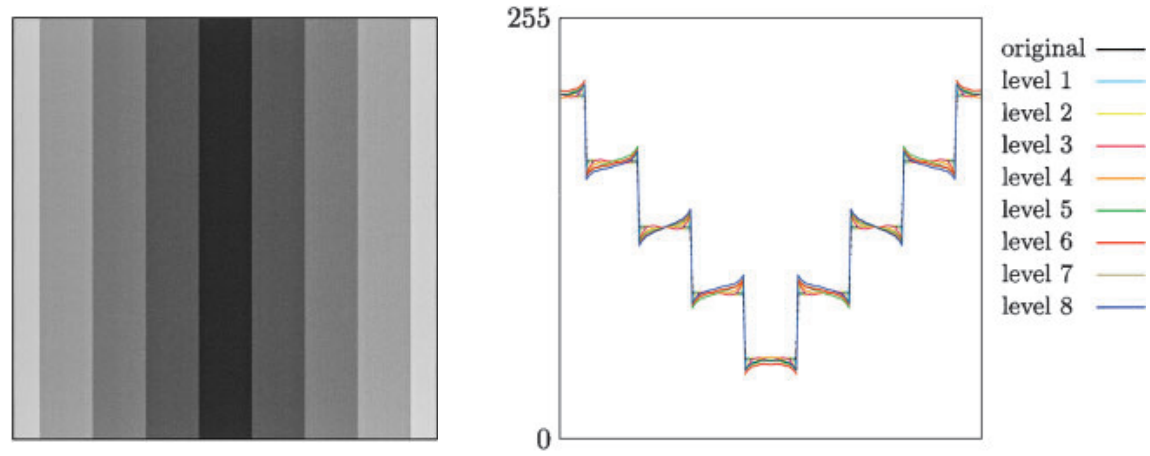

Fig. 12.
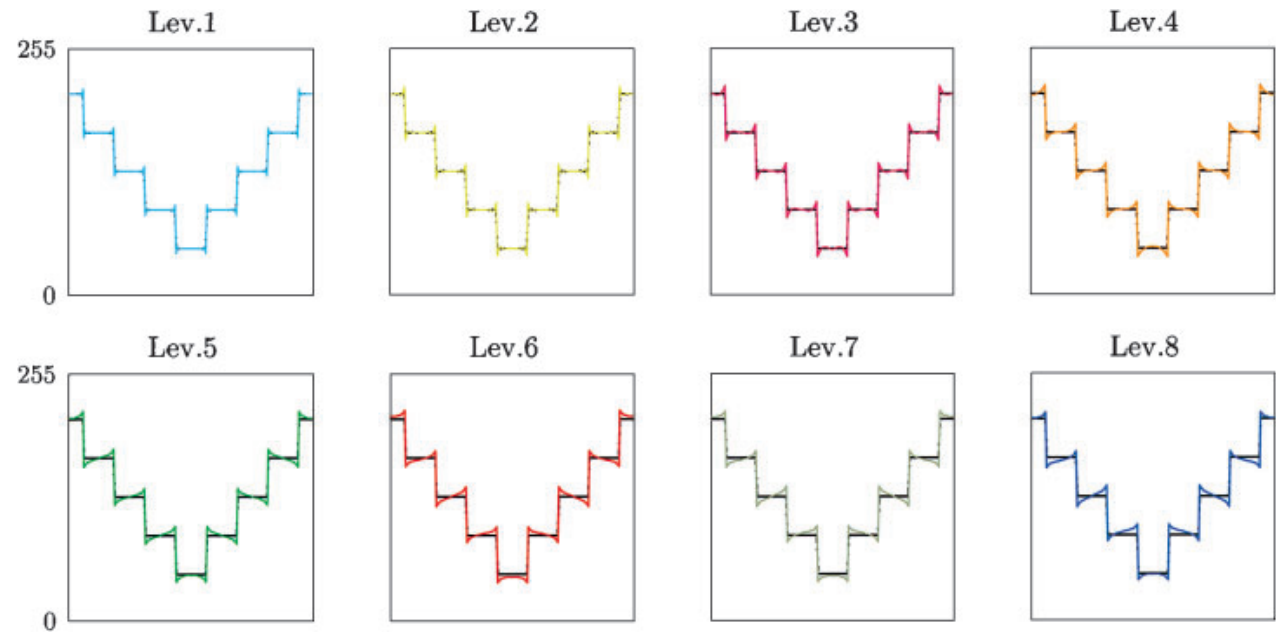

Fig. 13 .

used in this paper, the vertical details must be affected by the variation of the luminance of vertical strips. However almost all contrast exponents of the vertical details are smaller than 1 (see Fig. 14), and the fluctuations of the vertical details are very small (see Fig. 15). Therefore the vertical details are enhanced by the nonlinear operation, and consequently the illusion appears. It should be noted that such processing by the SNW system is useful in order to indicate the existence of two adjacent areas of different luminosities.

Now I turn to an interesting phenomenon related to the Chevreul illusion. It gives an evidence which indicates the nonlinearity of information processing by human's visual system. See two images in Fig. 16. Our perception is that there is a narrow brighter line along the edge in the left figure, however there is physically no such a stripe. On the other hand, in the right figure the strength of such illusion is weakened ([24]). This perception implies that our visual information processing is never linear. The SNW system can simulate this nonlinear phenomenon (see Fig. 17). The reason is that because the energies of the vertical details of the left image in Fig. 16 are smaller than ones for the right image, and consequently the contrast exponents of the left image are smaller than that of the right image (see Fig. 18). 


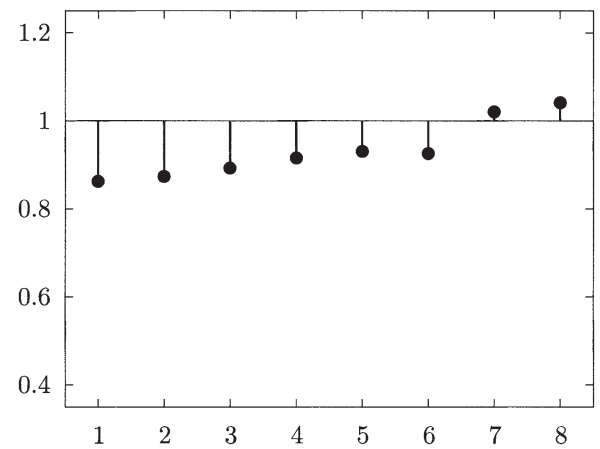

Fig. 14. Vertical contrast exponents of the Chevreul pattern.
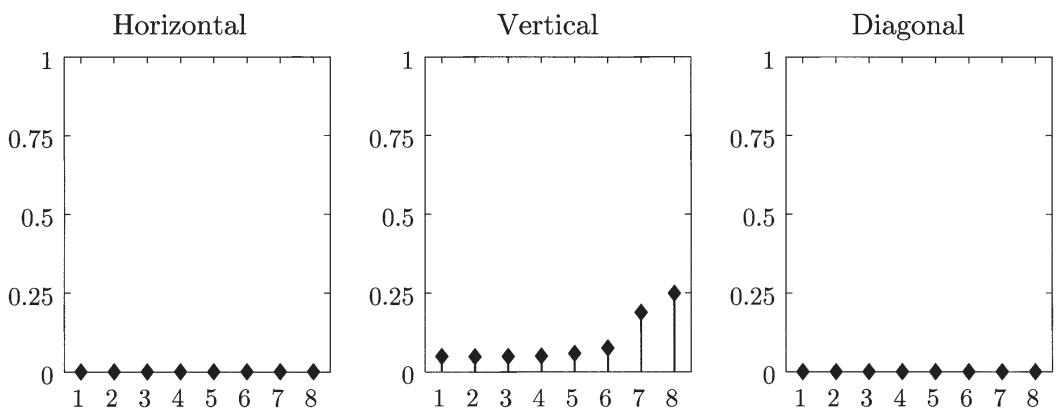

Fig. 15. Maxima of absolute values of $a b s\left(y_{k}^{\varepsilon}\right)$ for the Chevreul pattern.
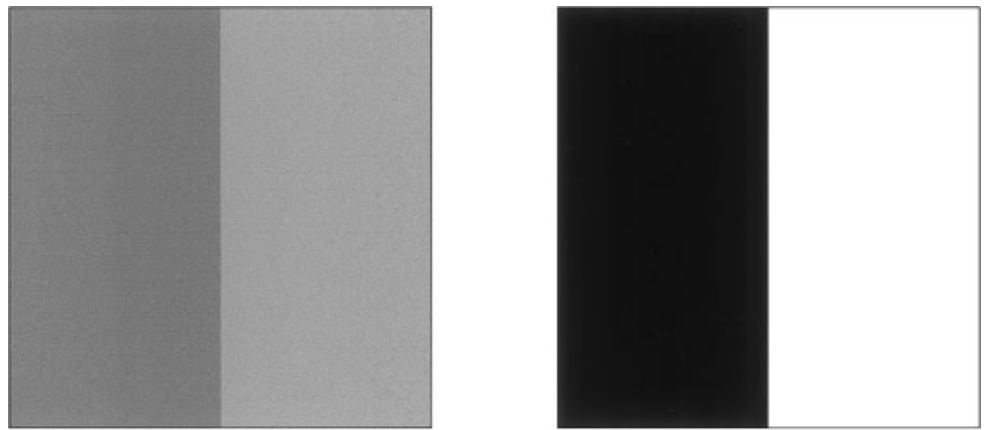

Fig. 16.
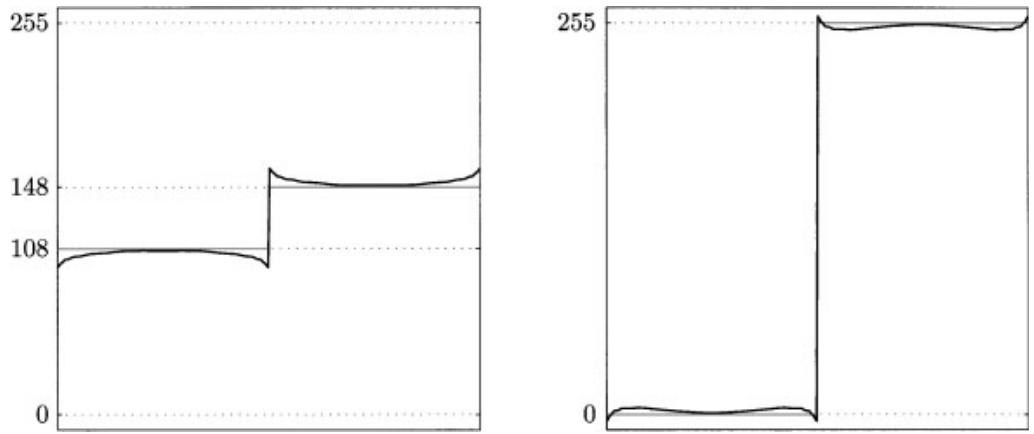

Fig. 17.

\subsection{The Mach band}

The left image of Fig. 19 is called the Mach band. In this image, bright and dark lines are perceived near the brighter and darker borders of blurred edges of the middle bright band. However physically the lines do not exist. In fact the right figure in Fig. 19 depicts the gray profile of the left image. It is well known that the occurrence of this illusion is explained by lateral inhibition effect by the retinal information processing. However also by use of the SNW system we can simulate this illusion. (See Fig. 20. The left image in Fig. 20 is scaled for displaying.) This means that the Mach 


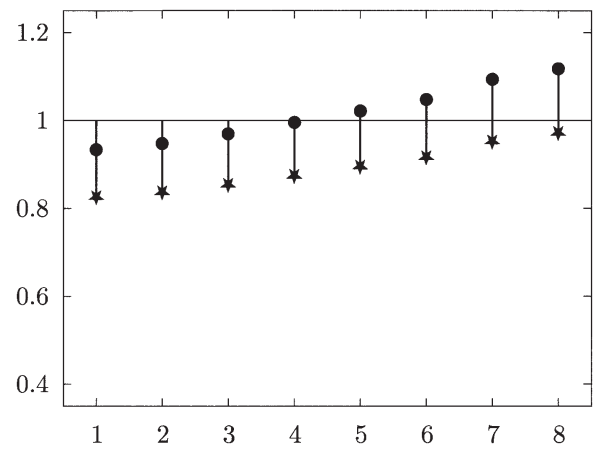

Fig. 18. Vertical contrast exponents for two bands in Fig. 16. ' $\star$ ': left image, '•’: right image.
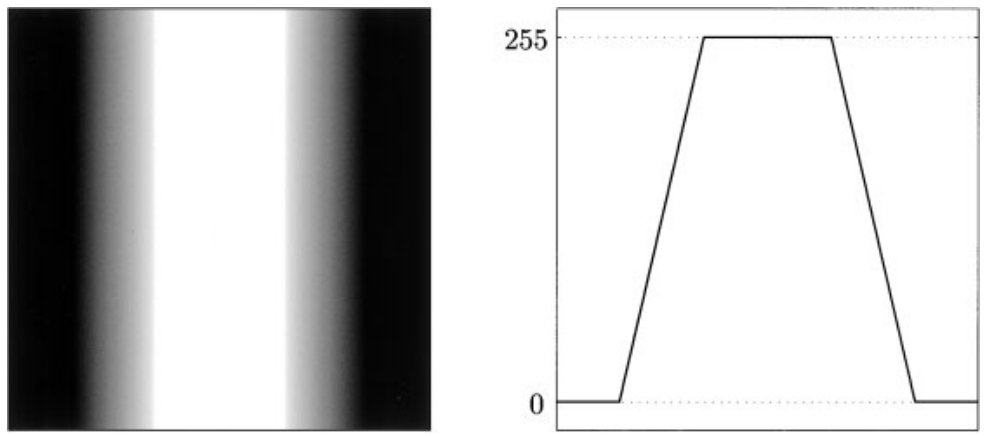

Fig. 19.
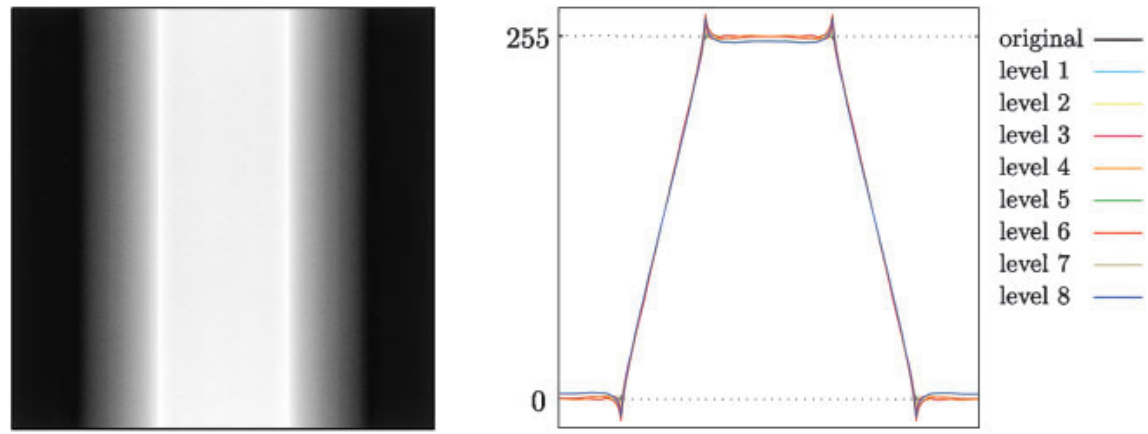

Fig. 20.

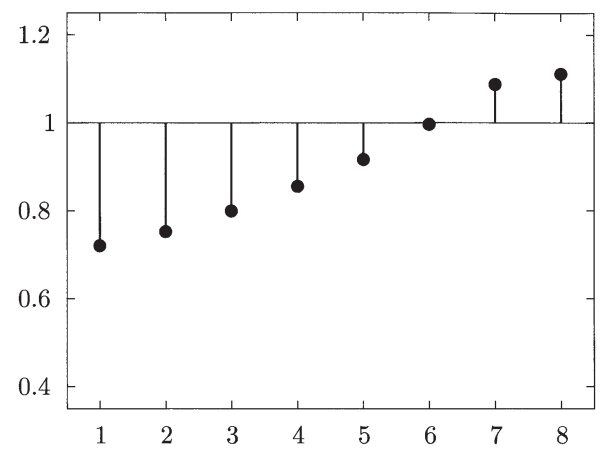

Fig. 21. Vertical contrast exponents for Fig. 19.

band illusion can be explained also by the contrast induction effect. Indeed the explanation is as follows: accurate calculation by computer implies that the $k$ th vertical contrast exponents $(k=1-6)$ are smaller than 1 (see Fig. 21$)$ and that the maxima of the values of $a b s\left(y_{k}^{v}\right)$ for $k=1-7$ are smaller than 0.5 (see Fig. 22). Therefore these vertical details are enhanced, and the illusion appears. 


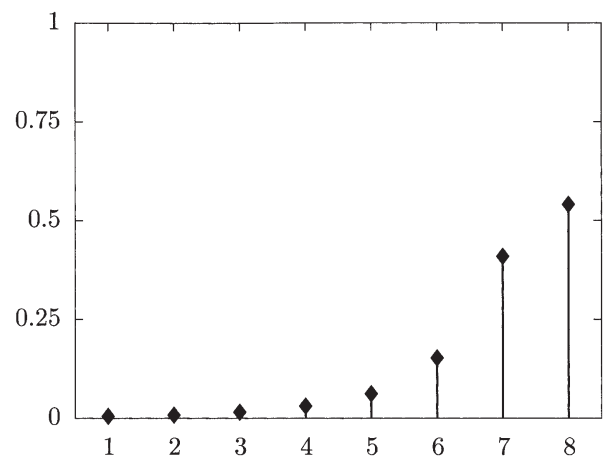

Fig. 22. Maxima of $a b s\left(y_{k}^{v}\right)$ for the Mach band.
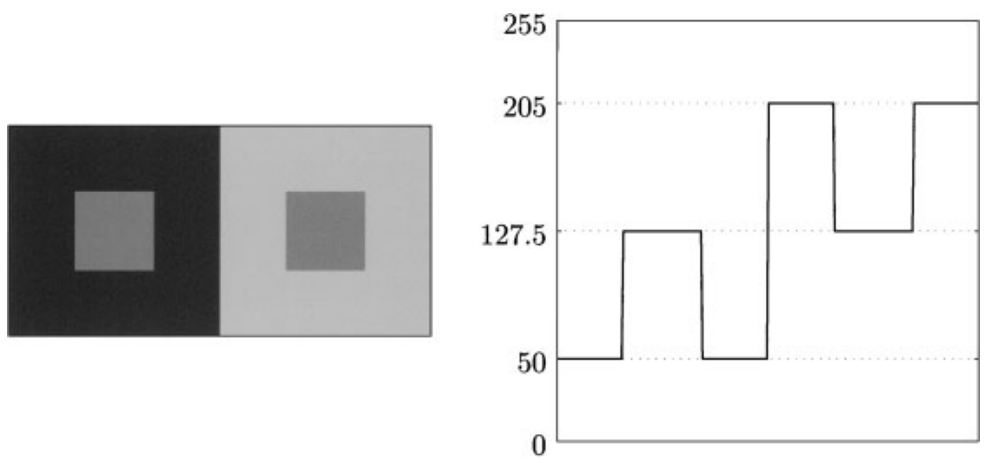

Fig. 23.
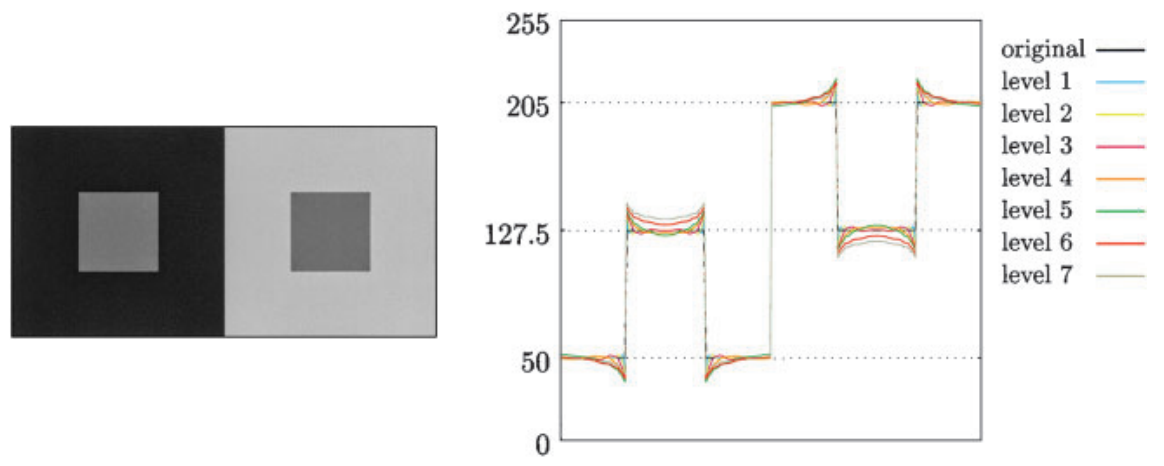

Fig. 24 .

\subsection{Simultaneous contrast and Todorovic's illusion}

The left image in Fig. 23 is classically well known as simultaneous brightness contrast illusion.

The luminance of the two gray inner squares is identical, however we perceive that the right inner square is darker than the left one. The result of the simulation in this paper is shown in Fig. 24. (Here the left square of the original image (the left of Fig. 23) and the right one are computed separately.) The reason why this illusion can be simulated is as follows. By computation all contrast exponents are smaller than 1, and the maxima of $a b s\left(y_{k}^{\varepsilon}\right)$ for all $\varepsilon \in\{h, v, d\}$ and for all $k$ are smaller than 0.5 . Therefore the details are enhanced, and consequently the edges of small squares are enhanced. Further at the 6th or 7th level, the luminance of the small square in the left output image (resp. the right output image) globally increases (resp. decreases), because the supports of the wavelets for that level spread out extensively.

Figure 25 is a lightness illusion shown by Todorovic ([27]). The inner small crosses in the right square and in the left square have same luminance, however the perception is that the right one is darker than the left one. As is asserted in [22, Chap. 3], the classical theory of lateral inhibition in the retina predicts logically that the left one must be darker than the right one. Computer simulation by the SNW system provides Fig. 26. In this output we can find a factor causing Todorovic's illusion. 

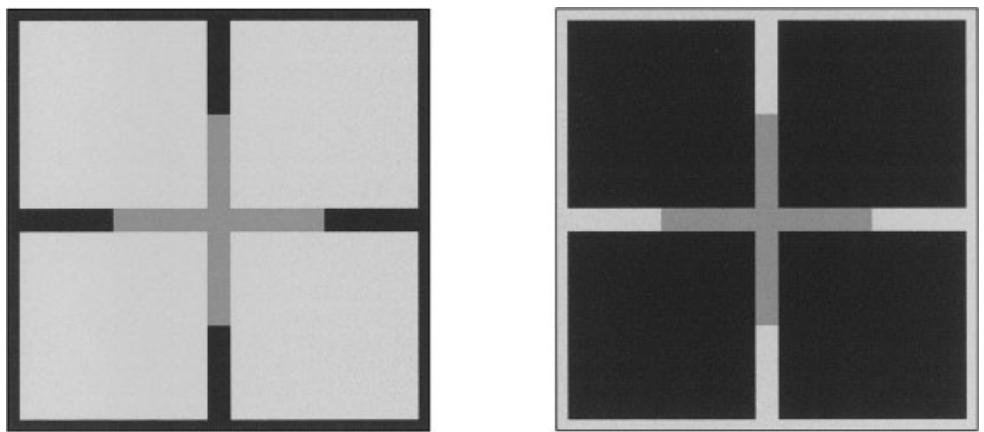

Fig. 25.
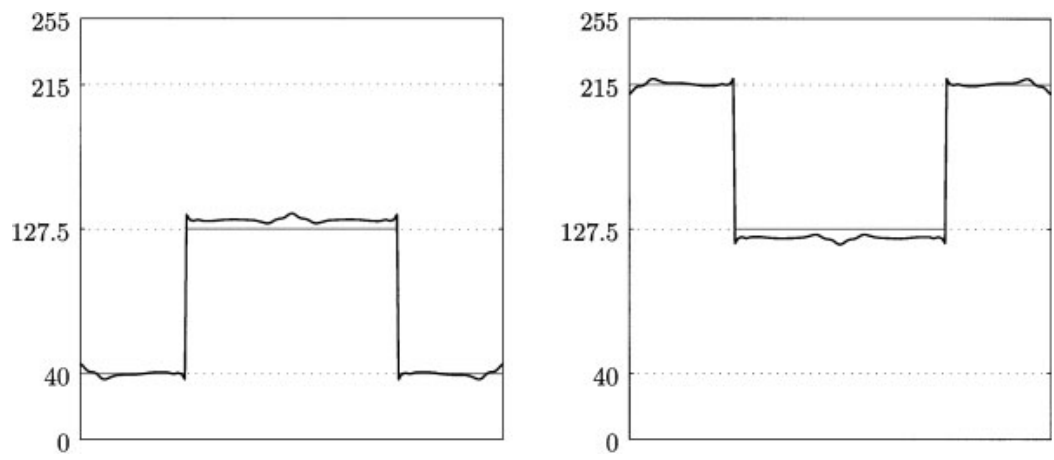

Fig. 26.

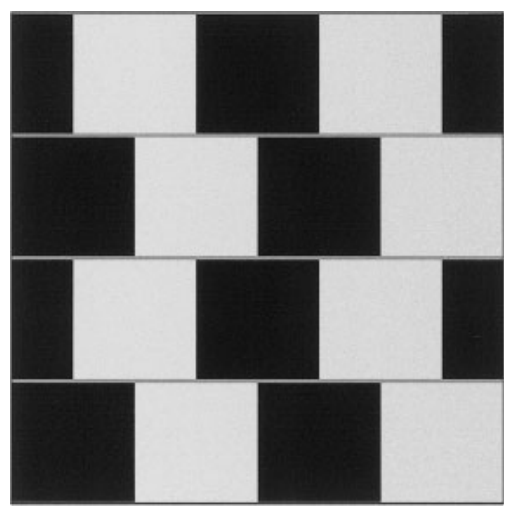

Fig. 27.

\section{The Café Wall Illusion and Its Variations}

Figure 27 is known as café wall illusion. The mortars in Fig. 27 are physically straight and parallel, but they are seen as tilted lines. This illusion was studied by Fraser (1908), Gregory and Heard (1979) et al. In particular, McCourt ([18]) and Earle and Maskell ([10]) explained the café wall illusion by using brightness induction and bandpass filtering respectively (see also Morgan and Moulden [20]). They showed that twisted cords are implicitly in the café wall pattern. Recently Kitaoka et al. gave a phenomenological explanation in terms of contrast polarities ([14]). There are also other studies about the café wall illusion. For a brief history of this illusion, see Kitaoka et al. [14].

In this paper this illusion is studied by using the SNW system. Applying the SNW system to the café wall pattern, the system produces the following output (Fig. 28).

Now let us show the picture of

$$
\widetilde{h}_{8} * F_{8}\left(x_{8}^{h}\right)-\widetilde{h}_{8} * x_{8}^{h}
$$

(see Fig. 29, which is scaled so that $[64,191]$ is mapped to $[0,255]$ ). In Fig. 29 there are some twisted cords which are a cause of the occurrence of the café wall illusion. These twisted cords are produced by the nonlinear processing modeled after our contrast induction. 


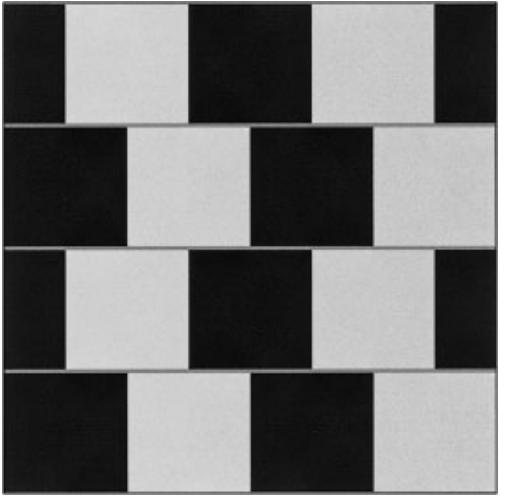

Fig. 28 .

(a)

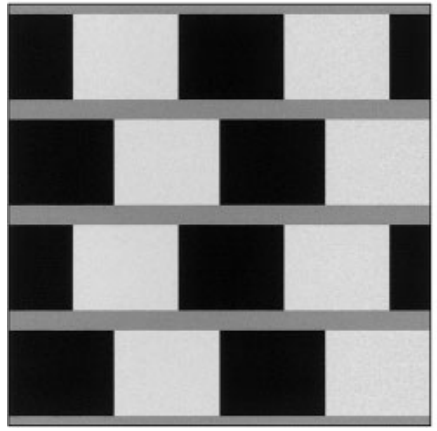

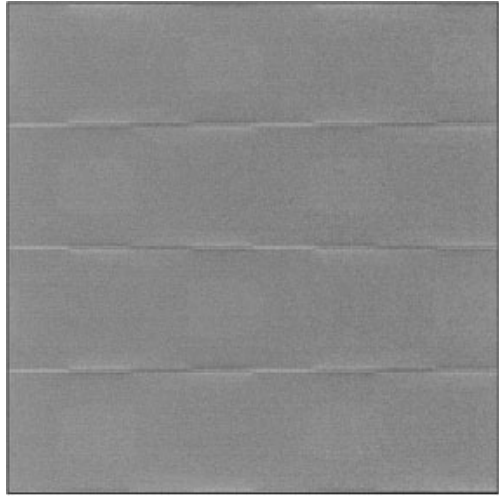

Fig. 29.

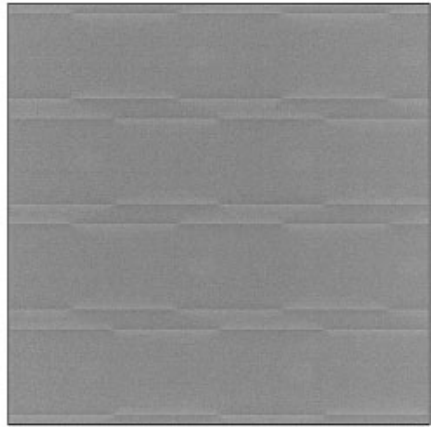

Fig. 30.

(a)

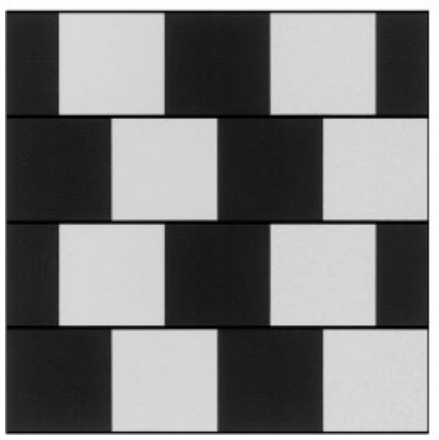

(b)

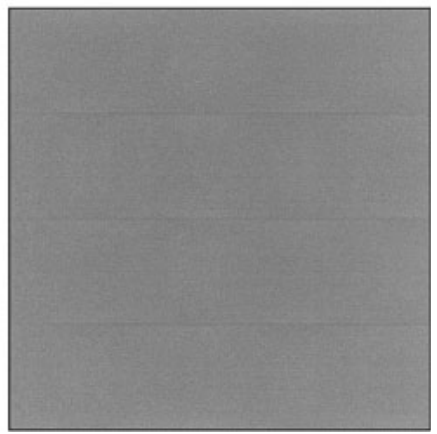

Fig. 31 .

It is known that if the mortars are wide (Fig. 30(a)) or are darker than the dark squares in the café wall pattern (Fig. 31(a)), the illusion disappears (see Gregory [13]). Indeed in these cases, (5) does not contain twisted cords as is shown in Fig. 30(b) for the former case, and in Fig. 31(b) for the latter case. In both cases, the images are scaled by the same way as Fig. 29.

\section{Natural Image}

In preceding sections several artificial patterns have been studied by using the SNW system. Here I deal with a natural image (Fig. 32, the upper left). If this natural image is inputted to the SNW system, then it outputs the image in the lower left of Fig. 32, which is scaled for displaying (see Section 4.1, for the definition of the scaling). The upper right (resp. the lower right) of Fig. 32 is a magnified picture of a part of the original natural image (resp. the outputted image). Based on this computer experiment, we can conclude that the SNW system has the ability to sharpen unclear parts of an image without sharpening unappropriately the original image. However this result is not unexpected, because the SNW system modeled itself on human's visual system which has such a function. 

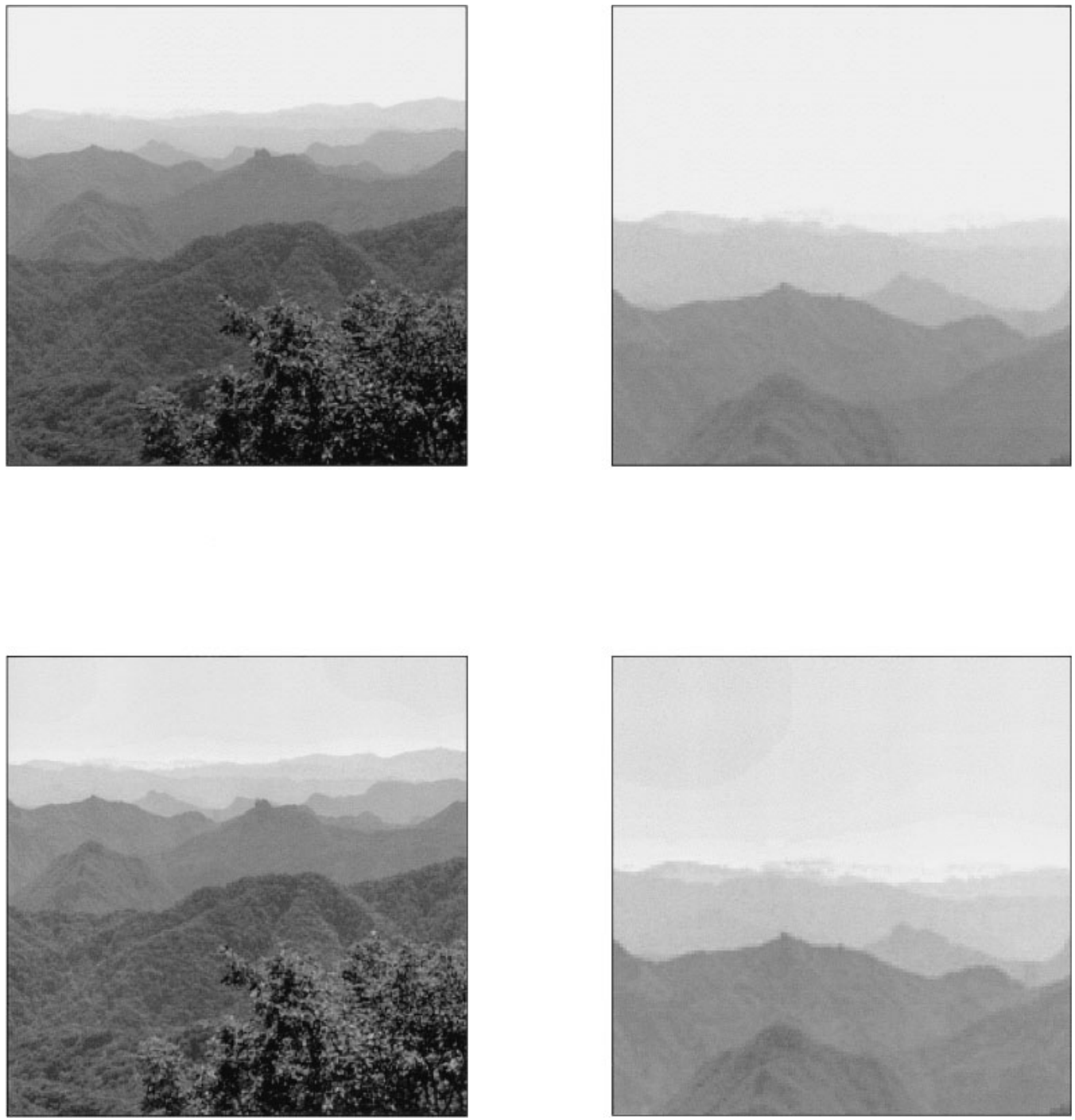

Fig. 32.

\section{Conclusion}

As described in Section 1, human's visual system enhances a contrast if it is surrounded by low contrast patterns, and inhibits it if it is surrounded by high contrast patterns. This is called the contrast induction. I proposed a mathematical description of the contrast induction by introducing the SN-function. Furthermore by incorporating this mathematical model of the contrast induction and a maximal overlap biorthogonal wavelet filter bank possessing some orientation selectivity, I constructed a computational nonlinear system, the SNW system. By this SNW system one can simulate the occurrence of various lightness illusions. As is well known, some lightness illusions cannot be explained by the classical theory of the lateral inhibition in the retina, and it is now believed that some postretinal processings also contribute lightness illusions. The simulations presented in this paper support mathematically that assertion.

As mentioned in Section 2, the simple cells have various oriented selectivity. A foreseeable extension of the study in this paper would be to implement the contrast induction model proposed here to an appropriate wavelet frame possessing many orientation selectivity.

\section{REFERENCES}

[1] Arai, H., Wavelets and vision (in Japanese), Sugaku no Tanosimi 2004 Aki (Have Fun with Mathematics 2004 Autum), Nihonhyouronsya, 78-99 (2004).

[2] Blakemore, S. J., and Campbell, F. W., "On the existence of neurons in the human visual system selectively responsive to the orientation and size of retinal images," J. Physiol., 203: 237-260 (1969).

[3] Blakeslee, B., and McCourt, M. E., "Similar mechanisms underlie simultaneous brightness contrast and grating induction," Vision Research, 37: 2849-2869 (1997).

[4] Blakeslee, B., and McCourt, M. E., "A multiscale spatial filtering account of the white effect, simultaneous brightness contrast and grating induction," Vision Research, 39: 4361-4377 (1999). 
[5] Campbell, F. W., and Robson, J. G., “Applications of Fourier analysis to the visibility of gratings,” J. Physiol., 197: 551-566 (1968).

[6] Cohen, A., Daubechies, I., and Feauveau, J.-C., "Biorthogonal bases of compactly supported wavelets," Comm. Pure and Appl. Math., 45: 485-560 (1992).

[7] Coifman, R. R., and Donoho, D. L., “Translation invariant de-noising,” Lecture Notes in Statistics, Vol. 103: pp. 125-150 (1995).

[8] Chubb, C. G., Sperling, G., and Solomon, J. A., "Texture interactions determine perceived contrast," Proc. Natl. Acad. Sci. U.S.A., 86: 9631-9635 (1989).

[9] De Valois, R. L., and De Valois, K. K., Spatial Vision, Oxford Univ. Press (1988).

[10] Earle, D. C., and Maskell, S. J., "Fraser cords and reversal of the café wall illusion," Perception, 22: $383-390$ (1993).

[11] Fiorentini, A., "Brightness and lightness," The Visual Neurosciences vol. 2 (L. M. Chalupa and J. S. Werner eds.), MIT Press, 881-891 (2004).

[12] Geisler, W. S., and Albrecht, D. G., Spatial Vision, Seeing (K. K. De Valois ed.), Academic Press, 79-128 (2000).

[13] Gregory, R. L., Eye and Brain, the Psychology of Seeing, 5th ed., Oxford Univ. Press (1998).

[14] Kitaoka, A., Pinna, B., and Brelstaff, G., "Contrast polarities determine the direction of Café Wall tilts," Perception, 33: 11-20 (2004).

[15] Lulich, D. P., and Stevens, K. A., "Differential contributions of circular and elongated spatial filters to the Café Wall illusion," Biological Cybernetics, 61: 427-435 (1989).

[16] McArther, J. A., and Moulden, B., "A two-dimensional model of brightness perception based on spatial filtering consistent with retinal processing," Vision Research, 39: 1199-1219 (1999).

[17] McCourt, M. E., “A spatial frequency dependent grating-induction effect," Vision Research, 22: 119-134 (1982).

[18] McCourt, M. E., "Brightness induction and the Café Wall illusion," Perception, 12: 131-142 (1983).

[19] Misiti, M., Misiti, Y., Oppenheim, G., and Poggi, J.-M., Wavelet Toolbox, Ver. 3, The MathWorks (2005).

[20] Morgan, M. J., and Moulden, B., “The Münsterberg figure and twisted cords," Vision Research, 26: 1793-1800 (1986).

[21] Nason, G. P., and Silverman, B. W., "The stationary wavelet transform and some statistical applications," Lecture Notes in Statistics, Vol. 103: 281-299 (1995).

[22] Palmer, S. E., Vision Science, Photons to Phenomenology, MIT Press (1999).

[23] Percival, D. B., and Walden, A. T., Wavelet Methods for Time Series Analysis, Cambridge Univ. Press (2000).

[24] Ratliff, F., "Why Mach bands are not seen at the edges of a step," Vision Research, 24: 163-166 (1984).

[25] Ross, J. R., Morrone, C., and Burr, D. C., “The conditions under which Mach bands are visible,” Vision Research, 29: 699-715 (1989).

[26] Spillmann, L., “The Hermann grid illusion: a tool for studying human perceptive field organization,” Perception, 23: 691-708 (1994).

[27] Todorovic, D., "Lightness and junctions," Perception, 26: 379-394 (1997).

[28] Wolfe, J. M., "Global factors in the Hermann grid illusion," Perception, 13: 33-40 (1984).

[29] Zeki, S., "The visual image in mind and brain," Scientific American (1992). 